\title{
On Axially Symmetric Vibrations of Fluid Filled Poroelastic Spherical Shells
}

\author{
Syed Ahmed Shah ${ }^{1}$, Mohammed Tajuddin ${ }^{2}$ \\ ${ }^{1}$ Department of Mathematics, Deccan College of Engineering and Technology, Hyderabad, India \\ ${ }^{2}$ Department of Mathematics, Osmania University, Hyderabad, India \\ E-mail:ahmed_shah67@yahoo.com \\ Received July 27, 2011; revised August 15, 2011; accepted August 19, 2011
}

\begin{abstract}
Employing Biot's theory of wave propagation in liquid saturated porous media, waves propagating in a hollow poroelastic closed spherical shell filled with fluid are studied. The frequency equation of axially symmetric vibrations for a pervious and an impervious surface is obtained. Free vibrations of a closed spherical shell are studied as a particular case when the fluid is vanished. Frequency as a function of ratio of thickness to inner radius is computed in absence of dissipation for two types of poroelastic materials each for a pervious and an impervious surface. Results of previous works are obtained as a particular case of the present study.
\end{abstract}

Keywords: Biot's Theory, Axially Symmetric Vibrations, Radial Vibrations, Rotatory Vibrations, Spherical Shell, Elastic Fluid, Pervious Surface, Impervious Surface, Frequency

\section{Introduction}

Using exact three dimensional equations of linear elasticity, Ram Kumar [1] studied the axially symmetric vibrations of fluid-filled spherical shells. Rand and DiMaggio [2] studied the vibrations of fluid filled elastic spherical and spheroidal shells. Employing Biot's [3] theory, Paul [4] discussed the radial vibrations of poroelastic spherical shells. Chao et al. [5] presented the theoretical and experimental results regarding wave propagation in porous formations. Ahmed Shah [6] investigated the axially symmetric vibrations of fluid-filled poroelastic circular cylindrical shells of various wall-thicknesses in the absence of dissipation. Sharma and Sharma [7] studied the three dimensional free vibrations of transradially thermoelastic spheres. Ahmed Shah and Tajuddin [8] studied torsional vibrations of poroelastic spheroidal shells.

In the present analysis, wave propagation in fluid filled poroelastic spherical shells is studied in absence of dissipation. Frequency equation each for a pervious and an impervious surface is obtained employing Biot's [3] theory of wave propagation in liquid saturated poroelastic solid. Biot's [3] model consists of an elastic matrix permeated by a network of interconnected spaces saturated with liquid. Frequency equation of axially symmet- ric vibrations each for a pervious and impervious surface is obtained for fluid filled and empty poroelastic spherical shells. The frequency equations of radial and rotatory vibrations are obtained as a particular case. Nondimensional frequency as a function of ratio of thickness to inner radius is computed for pervious and impervious surfaces in each case, i.e., fluid filled and empty poroelastic spherical shell in absence of dissipation. The results are displayed graphically for two types of poroelastic materials and then discussed. Results of some previous works are shown as a particular case of the present investigation. By ignoring the liquid effects, and after some rearrangement of terms, results of purely elastic solid are shown as a particular case considered by Ram Kumar [1].

\section{Governing Equations}

The equations of motion of a homogeneous, isotropic poroelastic solid [3] in presence of dissipation $b$ are

$$
\begin{aligned}
& P \nabla^{2} \Phi_{1}+Q \nabla^{2} \Phi_{2}=\frac{\partial^{2}}{\partial \mathrm{t}^{2}}\left(\rho_{11} \Phi_{1}+\rho_{12} \Phi_{2}\right)+b \frac{\partial}{\partial \mathrm{t}}\left(\Phi_{1}-\Phi_{2}\right), \\
& Q \nabla^{2} \Phi_{1}+R \nabla^{2} \Phi_{2}=\frac{\partial^{2}}{\partial \mathrm{t}^{2}}\left(\rho_{12} \Phi_{1}+\rho_{22} \Phi_{2}\right)-b \frac{\partial}{\partial \mathrm{t}}\left(\Phi_{1}-\Phi_{2}\right),
\end{aligned}
$$




$$
\begin{aligned}
& N \nabla^{2} \Psi_{1}=\frac{\partial^{2}}{\partial \mathrm{t}^{2}}\left(\rho_{11} \Psi_{1}+\rho_{12} \Psi_{2}\right)+b \frac{\partial}{\partial \mathrm{t}}\left(\Psi_{1}-\Psi_{2}\right), \\
& 0=\frac{\partial^{2}}{\partial \mathrm{t}^{2}}\left(\rho_{12} \Psi_{1}+\rho_{22} \Psi_{2}\right)-b \frac{\partial}{\partial \mathrm{t}}\left(\Psi_{1}-\Psi_{2}\right),
\end{aligned}
$$

where $\Phi_{1}, \Phi_{2}, \Psi_{1}, \Psi_{2}$ are potential functions of $r, \theta$ and time $t ; P(=A+2 N), N, Q, R$ are all poroelastic constants and $\rho_{i k}(i, k=1,2)$ are the mass coefficients following Biot [3]. The poroelastic constants $A, N$ correspond to familiar Lame' constants in purely elastic solid. The coefficient $\mathrm{N}$ represents the shear modulus of the solid. The coefficient $\mathrm{R}$ is a measure of the pressure required on the liquid to force a certain amount of the liquid into the aggregate while total volume remains constant. The coefficient $Q$ represents the coupling between the volume change of the solid to that of liquid and

$$
\nabla^{2}=\frac{\partial^{2}}{\partial r^{2}}+\frac{1}{r^{2}} \frac{\partial^{2}}{\partial \theta^{2}}+\frac{2}{r} \frac{\partial}{\partial r}+\frac{\cot \theta}{r^{2}} \frac{\partial}{\partial \theta}
$$

The equation of motion for a homogeneous, isotropic, inviscid elastic fluid is

$$
\nabla^{2} \Phi=\frac{1}{V_{\mathrm{f}}^{2}} \frac{\partial^{2} \Phi}{\partial t^{2}}
$$

where $\Phi$ is displacement potential function and $V_{f}$ is the velocity of sound in the fluid.

The stresses $\sigma_{i k}$ and the liquid pressure s of the poroelastic solid are

$$
\begin{gathered}
\sigma_{i k}=2 N e_{i k}+(A e+Q \in) \delta_{i k}, \quad(i, k=r, \theta, \varphi) \\
s=Q e+R \in,
\end{gathered}
$$

where $\delta_{\mathrm{ik}}$ is the well-known Kronecker delta function.

The fluid pressure $\sigma_{\mathrm{f}}$ is given by

$$
\sigma_{f}=-\rho_{f} \frac{\partial^{2} \Phi}{\partial t^{2}}
$$

where $\rho_{f}$ is the density of the fluid.

\section{Solution of the Problem}

Let $(r, \theta, \varphi)$ be the spherical polar coordinates. Consider a homogeneous, isotropic, poroelastic spherical shell filled with inviscid elastic fluid. Let the inner and outer radii be $r_{1}$ and $r_{2}$ respectively so that the thickness of poroelastic spherical shell is $h\left[=\left(r_{2}-r_{1}\right)>0\right]$. Axially sym- metric vibrations of the spherical shell is characterized by the displacement field of solid $\vec{u}=(u, v, 0)$ and liquid $\vec{U}=(U, V, 0)$ where $u, v, U$ and $V$ are functions of $r, \theta$ and time $t$. For axially symmetric vibrations of poroelastic spherical shell, the displacement components and solid and liquid, respectively are given potential functions $\Phi_{1}, \Phi_{2}, \Psi_{1}, \Psi_{2}$ as

$$
\begin{gathered}
u=\frac{\partial \Phi_{1}}{\partial r}+\frac{1}{r} \frac{\partial^{2} \Psi_{1}}{\partial \theta^{2}}+\frac{\cot \theta}{r} \frac{\partial \Psi_{1}}{\partial \theta}, \\
\mathrm{v}=\frac{1}{r} \frac{\partial \Phi_{1}}{\partial \theta}-\frac{\partial^{2} \Psi_{1}}{\partial r \partial \theta}-\frac{1}{r} \frac{\partial \Psi_{1}}{\partial \theta}, \\
U=\frac{\partial \Phi_{2}}{\partial r}+\frac{1}{r} \frac{\partial^{2} \Psi_{2}}{\partial \theta^{2}}+\frac{\cot \theta}{r} \frac{\partial \Psi_{2}}{\partial \theta}, \\
\mathrm{V}=\frac{1}{r} \frac{\partial \Phi_{2}}{\partial \theta}-\frac{\partial^{2} \Psi_{2}}{\partial r \partial \theta}-\frac{1}{r} \frac{\partial \Psi_{2}}{\partial \theta} .
\end{gathered}
$$

Similarly, the displacement vector of fluid is $\vec{u}_{f}=\left(u_{f}, v_{f}, 0\right)$, where

$$
u_{f}=\frac{\partial \Phi}{\partial r}, v_{f}=\frac{1}{r} \frac{\partial \Phi}{\partial \theta} .
$$

Solution of Equation (1) is

$$
\begin{gathered}
\Phi_{1}=\left[A_{1} j_{n}\left(\xi_{1} r\right)+B_{1} y_{n}\left(\xi_{1} r\right)+A_{2} j_{n}\left(\xi_{2} r\right)+B_{2} y_{n}\left(\xi_{2} r\right)\right] P_{n}(\cos \theta) \mathrm{e}^{i \omega t}, \\
\Phi_{2}=-\left[A_{1} \delta_{1}^{2} j_{n}\left(\xi_{1} r\right)+B_{1} \delta_{1}^{2} y_{n}\left(\xi_{1} r\right)+A_{2} \delta_{2}^{2} j_{n}\left(\xi_{2} r\right)+B_{2} \delta_{2}^{2} y_{n}\left(\xi_{2} r\right)\right] P_{n}(\cos \theta) \mathrm{e}^{i \omega t}, \\
\Psi_{1}=\left[A_{3} j_{n}\left(\xi_{3} r\right)+B_{3} y_{n}\left(\xi_{3} r\right)\right] P_{n}(\cos \theta) \mathrm{e}^{i \omega t}, \quad \Psi_{2}=-\frac{K_{12}}{K_{22}}\left[A_{3} j_{n}\left(\xi_{3} r\right)+B_{3} y_{n}\left(\xi_{3} r\right)\right] P_{n}(\cos \theta) \mathrm{e}^{i \omega t} .
\end{gathered}
$$

In Equation (8), $j_{n}, y_{n}$ are Spherical Bessel functions of first and second kind respectively, $P_{n}$ is Legendre polynomial of degree $n$ ( $n$ is the order of spherical harmonic),
$A_{1}, B_{1}, A_{2}, B_{2}, A_{3}, B_{3}$ are constants, $\omega$ is circular frequency. Here $i$ is complex unity or $i^{2}=-1$ and

$$
\xi_{k}=\omega V_{\mathrm{k}}^{-1}, k=1,2,3
$$


In Equation (9), $V_{1}, V_{2}$ are the dilatational wave velocities of first and second kind, respectively and $V_{3}$ is shear wave velocity.

A bounded solution of Equation (3) is

$$
\Phi=A_{f} j_{n}\left(\xi_{f} r\right) P_{n}(\cos \theta) \mathrm{e}^{i \omega t},
$$

where $A_{\mathrm{f}}$ is constant and

$$
\begin{gathered}
\xi_{f}=\omega V_{\mathrm{f}}^{-1} . \\
u-u_{f}=\left[A_{1} M_{11}(r)+\mathrm{B}_{1} M_{12}(r)+A_{2} M_{13}(r)+B_{2} M_{14}(r)+A_{3} M_{15}(r)+B_{3} M_{16}(r)+A_{\mathrm{f}} M_{17}(r)\right] P_{n}(\cos \theta) \mathrm{e}^{i \omega t}, \\
\sigma_{r r}+s-\sigma_{f}=\left[A_{1} M_{21}(r)+B_{1} M_{22}(r)+A_{2} M_{23}(r)+B_{2} M_{24}(r)+A_{3} M_{25}(r)+B_{3} M_{26}(r)+A_{\mathrm{f}} M_{27}(r)\right] \mathrm{P}_{\mathrm{n}}(\cos \theta) \mathrm{e}^{i \omega t}, \\
\sigma_{r \theta}=\left[A_{1} M_{31}(r)+B_{1} M_{32}(r)+A_{2} M_{33}(r)+B_{2} M_{34}(r)+A_{3} M_{35}(r)+B_{3} M_{36}(r)\right] \frac{\mathrm{d} P_{n}(\cos \theta)}{\mathrm{d} \theta} \sin \theta \mathrm{e}^{i \omega t}, \\
s-\sigma_{f}=\left[A_{1} M_{41}(r)+B_{1} M_{42}(r)+A_{2} M_{43}(r)+B_{2} M_{44}(r)+A_{\mathrm{f}} M_{47}(r)\right] P_{n}(\cos \theta) \mathrm{e}^{i \omega t}, \\
\frac{\partial s}{\partial r}-\frac{\partial \sigma_{f}}{\partial r}=\left[A_{1} N_{41}(r)+B_{1} N_{42}(r)+A_{2} N_{43}(r)+B_{2} N_{44}(r)+A_{\mathrm{f}} N_{47}(r)\right] P_{n}(\cos \theta) \mathrm{e}^{i \omega t},
\end{gathered}
$$

where the elements $M_{i k}(r)$ and $N_{i k}(r)$ are

$$
\begin{gathered}
M_{11}(r)=\xi_{1} j_{n}^{\prime}\left(\xi_{1} r\right), M_{12}(r)=\xi_{1} y_{n}^{\prime}\left(\xi_{1} r\right), M_{13}(r)=\xi_{2} j_{n}^{\prime}\left(\xi_{2} r\right), M_{14}(r)=\xi_{2} y_{n}^{\prime}\left(\xi_{2} r\right), \\
M_{15}(r)=-\frac{n(n+1)}{r} j_{n}\left(\xi_{3} r\right), M_{16}(r)=-\frac{n(n+1)}{r} y_{n}\left(\xi_{3} r\right), M_{17}(r)=-\xi_{f} j_{n}^{\prime}\left(\xi_{f} r\right), \\
M_{21}(r)=\left(P+Q-Q \delta_{1}^{2}-R \delta_{1}^{2}\right) \xi_{1}^{2} j_{n}^{\prime \prime}\left(\xi_{1} r\right)+\left(A+Q-Q \delta_{1}^{2}-R \delta_{1}^{2}\right) \frac{2 \xi_{1}}{r} j_{n}^{\prime}\left(\xi_{1} r\right)-\left(A+Q-Q \delta_{1}^{2}-R \delta_{1}^{2}\right) \frac{n(n+1)}{r^{2}} j_{n}\left(\xi_{1} r\right) \\
M_{22}(r)=\left(P+Q-Q \delta_{1}^{2}-R \delta_{1}^{2}\right) \xi_{1}^{2} y_{\mathrm{n}}^{\prime \prime}\left(\xi_{1} r\right)+\left(A+Q-Q \delta_{1}^{2}-R \delta_{1}^{2}\right) \frac{2 \xi_{1}}{r} y_{\mathrm{n}}^{\prime}\left(\xi_{1} r\right)-\left(A+Q-Q \delta_{1}^{2}-R \delta_{1}^{2}\right) \frac{n(n+1)}{r^{2}} y_{n}\left(\xi_{1} r\right), \\
M_{23}(r)=\left(P+Q-Q \delta_{2}^{2}-R \delta_{2}^{2}\right) \xi_{2}^{2} j_{n}^{\prime \prime}\left(\xi_{2} r\right)+\left(A+Q-Q \delta_{2}^{2}-R \delta_{2}^{2}\right) \frac{2 \xi_{2}}{r} j_{\mathrm{n}}^{\prime}\left(\xi_{2} r\right)-\left(A+Q-Q \delta_{2}^{2}-R \delta_{2}^{2}\right) \frac{n(n+1)}{r^{2}} j_{n}\left(\xi_{2} r\right), \\
M_{24}(r)=\left(P+Q-Q \delta_{2}^{2}-R \delta_{2}^{2}\right) \xi_{2}^{2} \mathrm{y}_{\mathrm{n}}^{\prime \prime}\left(\xi_{2} r\right)+\left(A+Q-Q \delta_{2}^{2}-R \delta_{2}^{2}\right) \frac{2 \xi_{2}}{r} y_{n}^{\prime}\left(\xi_{2} r\right)-\left(A+Q-Q \delta_{2}^{2}-R \delta_{2}^{2}\right) \frac{n(n+1)}{r^{2}} y_{n}\left(\xi_{2} r\right) \\
M_{25}(r)=-\frac{2 N n(n+1) \xi_{3}}{r} j_{\mathrm{n}}^{\prime}\left(\xi_{3} r\right)+\frac{2 N n(n+1)}{r^{2}} j_{n}\left(\xi_{3} r\right), \\
M_{26}(r)=-\frac{2 N n(n+1) \xi_{3}}{r} y_{n}^{\prime}\left(\xi_{3} r\right)+\frac{2 N n(n+1)}{r^{2}} y_{n}\left(\xi_{3} r\right), M_{27}(r)=-\rho_{f} w^{2} j_{n}\left(\xi_{f} r\right), \\
M_{31}(r)=-\frac{2 N \xi_{1}}{r} j_{n}^{\prime}\left(\xi_{1} r\right)+\frac{2 N}{r^{2}} j_{n}\left(\xi_{1} r\right), M_{32}(r)=-\frac{2 N \xi_{1}}{r} y_{n}^{\prime}\left(\xi_{1} r\right)+\frac{2 N}{r^{2}} y_{n}\left(\xi_{1} r\right), \\
M_{33}(r)=-\frac{2 N \xi_{2}}{r} j_{n}^{\prime}\left(\xi_{2} r\right)+\frac{2 N}{r^{2}} j_{n}\left(\xi_{2} r\right), M_{34}(r)=-\frac{2 N \xi_{2}}{r} y_{n}^{\prime}\left(\xi_{2} r\right)+\frac{2 N}{r^{2}} y_{n}\left(\xi_{2} r\right), \\
M_{41}(r)=\left(Q-R \delta_{1}^{2}\right)\left[\xi_{1}^{2} j_{n}^{\prime \prime}\left(\xi_{1} r\right)+\frac{2 \xi_{1}}{r} j_{n}^{\prime}\left(\xi_{1} r\right)-\frac{n(n+1)}{r^{2}} j_{n}\left(\xi_{1} r\right)\right] \\
M_{35}(r)=\mathrm{N} \xi_{3}^{2} j_{\mathrm{n}}^{\prime \prime}\left(\xi_{3} \mathrm{r}\right)+\frac{N n(n+1)-2 N}{r^{2}} j_{n}\left(\xi_{3} r\right), M_{36}(r)=N \xi_{3}^{2} y_{\mathrm{n}}^{\prime \prime}\left(\xi_{3} r\right)+\frac{N n(n+1)-2 N}{r^{2}} y_{n}\left(\xi_{3} r\right),
\end{gathered}
$$




$$
\begin{gathered}
M_{42}(r)=\left(Q-R \delta_{1}^{2}\right)\left[\xi_{1}^{2} y_{\mathrm{n}}^{\prime \prime}\left(\xi_{1} r\right)+\frac{2 \xi_{1}}{r} y_{\mathrm{n}}^{\prime}\left(\xi_{1} r\right)-\frac{n(n+1)}{r^{2}} y_{n}\left(\xi_{1} r\right)\right] \\
M_{43}(r)=\left(Q-R \delta_{2}^{2}\right)\left[\xi_{2}^{2} j_{\mathrm{n}}^{\prime \prime}\left(\xi_{2} r\right)+\frac{2 \xi_{2}}{r} j_{n}^{\prime}\left(\xi_{2} r\right)-\frac{n(n+1)}{r^{2}} j_{n}\left(\xi_{2} r\right)\right] \\
M_{44}(r)=\left(Q-R \delta_{2}^{2}\right)\left[\xi_{2}^{2} y_{n}^{\prime \prime}\left(\xi_{2} r\right)+\frac{2 \xi_{2}}{r} y_{n}^{\prime}\left(\xi_{2} r\right)-\frac{n(n+1)}{r^{2}} y_{n}\left(\xi_{2} r\right)\right], \\
M_{45}(r)=0, M_{46}(r)=0, M_{47}(r)=-\rho_{f} \omega^{2} j_{n}\left(\xi_{f} r\right), \\
N_{41}(r)=\left(Q-R \delta_{1}^{2}\right)\left[\xi_{1}^{3} j_{\mathrm{n}}^{\prime \prime \prime}\left(\xi_{1} r\right)+\frac{2 \xi_{1}^{2}}{r} j_{\mathrm{n}}^{\prime \prime}\left(\xi_{1} r\right)-\frac{\left(n^{2}+n+2\right) \xi_{1}}{r^{2}} j_{\mathrm{n}}^{\prime}\left(\xi_{1} r\right)+\frac{2 n(n+1)}{r^{3}} j_{n}\left(\xi_{1} r\right)\right] \\
N_{42}(r)=\left(Q-R \delta_{1}^{2}\right)\left[\xi_{1}^{3} y_{n}^{\prime \prime \prime}\left(\xi_{1} r\right)+\frac{2 \xi_{1}^{2}}{r} \mathrm{y}_{\mathrm{n}}^{\prime \prime}\left(\xi_{1} r\right)-\frac{\left(n^{2}+n+2\right) \xi_{1}}{r^{2}} y_{\mathrm{n}}^{\prime}\left(\xi_{1} r\right)+\frac{2 n(n+1)}{r^{3}} y_{n}\left(\xi_{1} r\right)\right] \\
N_{43}(r)=\left(Q-R \delta_{2}^{2}\right)\left[\xi_{2}^{3} j_{n}^{\prime \prime \prime}\left(\xi_{2} r\right)+\frac{2 \xi_{2}^{2}}{r} j_{n}^{\prime \prime}\left(\xi_{2} r\right)-\frac{\left(n^{2}+n+2\right) \xi_{2}}{r^{2}} j_{n}^{\prime}\left(\xi_{2} \mathrm{r}\right)+\frac{2 n(n+1)}{r^{3}} j_{n}\left(\xi_{2} r\right)\right] \\
N_{44}(r)=\left(Q-R \delta_{2}^{2}\right)\left[\xi_{2}^{3} y_{n}^{\prime \prime \prime}\left(\xi_{2} r\right)+\frac{2 \xi_{2}^{2}}{r} y_{n}^{\prime \prime}\left(\xi_{2} r\right)-\frac{\left(n^{2}+n+2\right) \xi_{2}}{r^{2}} y_{n}^{\prime}\left(\xi_{2} r\right)+\frac{2 n(n+1)}{\mathrm{r}^{3}} y_{n}\left(\xi_{2} r\right)\right] \\
N_{45}(r)=0, N_{46}(r)=0, N_{47}(r)=-\rho_{f} \omega^{2} \xi_{f} j_{n}^{\prime}\left(\xi_{f} r\right) .
\end{gathered}
$$

In Equation (22), a prime over $j_{n}$ or $y_{n}$ represents differentiation with respect to $r$.

\section{Boundary Conditions-Frequency Equation}

The outer surface of the poroelastic spherical shell is assumed to be free from traction. Continuity of radial displacement and fluid pressure is assumed at the interface of solid and fluid. Thus the boundary conditions for a fluid-filled poroelastic spherical shell, in case of a pervious surface are

$$
\begin{gathered}
u-u_{f}=0, \sigma_{r r}+s-\sigma_{f}=0, \sigma_{r \theta}=0, s-\sigma_{f}=0 \quad \text { at } r=r_{1}, \\
\sigma_{r r}+s=0, \sigma_{r \theta}=0, s=0, \text { at } r=r_{2} .
\end{gathered}
$$

Similarly, the boundary conditions for a fluid-filled poroelastic spherical shell, in case of an impervious surface are

$$
\begin{aligned}
& u-u_{f}=0, \sigma_{r r}+\mathrm{s}-\sigma_{f}=0, \sigma_{r \theta}=0, \frac{\partial s}{\partial r}-\frac{\partial \sigma_{f}}{\partial r}=0, \\
& \text { at } r=r_{1}, \sigma_{r r}+s=0, \sigma_{r \theta}=0, \frac{\partial s}{\partial r}=0, \text { at } r=r_{2} .
\end{aligned}
$$

Substitution of Equations (17)-(20) into the boundary conditions (23) result in a system of seven homogeneous algebraic equations in seven constants $A_{1}, B_{1}, A_{2}, B_{2}, A_{3}$, $B_{3}$ and $A_{f}$. For a non-trivial solution, the determinant of the coefficients must vanish. By eliminating these constants, the frequency equation of axially symmetric vibrations of a fluid-filled poroelastic spherical shell in case of a pervious surface is

$$
\left|A_{i k}\right|=0, i, k=1,2,3, \cdots 7 .
$$

In Equation (25), the elements $A_{i k}$ are

$$
\begin{gathered}
A_{i k}=M_{i k}\left(r_{1}\right) ; \quad i=1,2,3,4 \text { and } k=1,2, \cdots 7, \\
A_{5 k}=M_{2 k}\left(r_{2}\right), A_{6 k}=M_{3 k}\left(r_{2}\right), A_{7 k}=M_{4 k}\left(r_{2}\right), k=1,2, \cdots 6, \\
A_{37}=0, \quad A_{57}=0, \quad A_{67}=0, A_{77}=0 .
\end{gathered}
$$

In Equation (26), the elements $M_{i k}(r)$ are defined in Equation (22).

Arguing on similar lines, Equations (17)-(19), (21) together with the Equation (24) yield the frequency equation of axially symmetric vibrations of a fluid-filled poroelastic spherical shell in case of an impervious surface to be

$$
\left|B_{i k}\right|=0, i, k=1,2, \cdots 7,
$$

where the elements $B_{i k}$ are

$$
B_{i k}=A_{i k}, i=1,2,3,5,6 \text { and } k=1,2, \cdots 7,
$$




$$
\begin{gathered}
B_{4 k}=N_{4 k}\left(r_{1}\right), \quad k=1,2, \cdots 7, \\
B_{7 \mathrm{k}}=\mathrm{N}_{4 \mathrm{k}}\left(r_{2}\right), k=1,2, \cdots 6, \text { and } B_{77}=0,
\end{gathered}
$$

where $M_{i k}(r)$ and $\mathrm{N}_{\mathrm{ik}}(r)$ are defined in Equation (22).

By eliminating liquid effects from frequency equation of a pervious surface (25), that is, setting $b \rightarrow 0, \rho_{12} \rightarrow 0$, $\rho_{22} \rightarrow 0,\left(A-Q^{2} / R\right) \rightarrow \lambda, N \rightarrow \mu, Q \rightarrow 0, R \rightarrow 0$ and after rearrangement of terms, the results of purely elastic solid are obtained as a particular case considered by Ram Kumar [1]. The frequency equation of an impervious surface (27) has no counterpart in purely elastic solid. The order of spherical harmonic $n$ takes different integer values. When $n=0$, radial vibrations are obtained whereas $n=1$ results in rotatory vibrations. For $n=2$ spheroidal vibrations are obtained in which the sphere is distorted into an ellipsoid of revolution becoming prolate or oblate according to the phase of motion.

\subsection{Frequency Equation for an Empty Poroelastic Spherical Shell}

When the fluid density is zero, that is, $\rho_{f}=0$ the fluidfilled poroelastic spherical shell will become an empty poroelastic spherical shell. Thus, the frequency equation of pervious surface (25) under suitable boundary conditions reduce to

$$
\left|A_{i k}\right|=0, i=2,3,4,5,6,7 \text { and } k=1,2,3,4,5,6,
$$

where the elements $A_{i k}$ are defined in Equation (26) are now evaluated for $\rho_{f}=0$.

Equation (29) is the frequency equation of axially symmetric vibrations of an empty poroelastic spherical shell in case of a pervious surface.

Similarly, the frequency equation of axially symmetric vibrations of an empty poroelastic spherical shell for an impervious surface under suitable boundary conditions is

$$
\left|B_{i k}\right|=0, i=2,3,4,5,6,7 \text { and } k=1,2,3,4,5,6,
$$

where the elements $B_{i k}$ are defined in Equation (28) are now evaluated for $\rho_{f}=0$.

\subsection{Frequency Equation of Radial Vibrations}

When the order of spherical harmonic $n=0$, the frequency equation of axially symmetric vibrations of fluid filled spherical shell Equation (25) reduce to the frequency equation of radial vibrations of fluid filled spherical shell in case of a pervious surface under suitable boundary conditions. Radial vibrations involve $u$ and $u_{f}$ as the only non-zero displacement components. Thus the frequency equation of radial vibrations of fluid filled poroelastic spherical shell for a pervious surface is

$$
\left|\begin{array}{ccccc}
A_{11} & A_{12} & A_{13} & A_{14} & A_{17} \\
A_{21} & A_{22} & A_{23} & A_{24} & A_{27} \\
A_{41} & A_{42} & A_{43} & A_{44} & A_{47} \\
A_{51} & A_{52} & A_{53} & A_{54} & 0 \\
A_{71} & A_{72} & A_{73} & A_{74} & 0
\end{array}\right|=0,
$$

where the elements $A_{i k}$ are defined in Equation (26) are now evaluated for $n=0$.

Similarly, the frequency equation of radial vibrations of fluid-filled poroelastic spherical shell in case of an impervious surface is

$$
\left|\begin{array}{lllll}
B_{11} & B_{12} & B_{13} & B_{14} & B_{17} \\
B_{21} & B_{22} & B_{23} & B_{24} & B_{27} \\
B_{41} & \mathrm{~B}_{42} & B_{43} & B_{44} & B_{47} \\
B_{51} & B_{52} & B_{53} & B_{54} & 0 \\
B_{71} & B_{72} & B_{73} & B_{74} & 0
\end{array}\right|=0,
$$

where the elements $B_{i k}$ are defined in Equation (28) are now evaluated for $n=0$.

When the fluid density is zero in Equation (31), it becomes the frequency equation of radial vibration of an empty poroelastic spherical shell under suitable boundary conditions as

$$
\left|\begin{array}{cccc}
A_{21} & A_{22} & A_{23} & A_{24} \\
A_{41} & A_{42} & A_{43} & A_{44} \\
A_{51} & A_{52} & A_{53} & A_{54} \\
A_{71} & A_{72} & A_{73} & A_{74}
\end{array}\right|=0,
$$

where the elements $A_{i k}$ are defined in Equation (26) are now evaluated for $\rho_{f}=0$ and $n=0$.

In a similar way, the frequency equation of radial vibrations of an empty poroelastic spherical shell for an impervious surface is

$$
\left|\begin{array}{llll}
B_{21} & B_{22} & B_{23} & B_{24} \\
B_{41} & B_{42} & B_{43} & B_{44} \\
B_{51} & B_{52} & B_{53} & B_{54} \\
B_{71} & B_{72} & B_{73} & B_{74}
\end{array}\right|=0
$$

where the elements $B_{i k}$ are defined in Equation (28) are now evaluated for $\rho_{f}=0$ and $n=0$.

The frequency equation of radial vibrations of an empty poroelastic spherical shell Equation (33) of a pervious surface was also studied by Paul [4] with the boundary conditions $\sigma_{r r}=0, s=0$, at $r=r_{1}$ and $r_{2}$.

\subsection{Frequency Equation of Rotatory Vibrations}

When the order of spherical harmonic $n=1$, the frequency equation of axially symmetric vibrations of fluid filled spherical shell Equation (25) reduce to the fre- 
quency equation of rotatory vibrations of fluid filled spherical shell in case of a pervious surface. Thus the frequency equation of rotatory vibrations of fluid filled poroelastic spherical shell for a pervious surface is

$$
\left|A_{i k}\right|=0, i, k=1,2,3, \cdots 7 .
$$

The elements $A_{i k}$ appearing in Equation (35) are defined in Equation (26) are now evaluated for $n=1$.

Similarly, the frequency equation of rotatory vibrations of a fluid filled poroelastic spherical shell in case of an impervious surface is

$$
\left|B_{i k}\right|=0, i, k=1,2,3, \cdots 7,
$$

where elements $B_{i k}$ appearing in Equation (36) are de- fined in Equation (28) are now evaluated for $n=1$.

Setting $n=1$ in Equations (29) and (30), the frequency equations of rotatory vibrations of empty poroelastic spherical shells of a pervious and an impervious surface respectively, are obtained.

\section{Non-Dimensionalisation of Frequency Equation}

To analyze the frequency equations of radial and rotatory vibrations of fluid-filled and empty poroelastic spherical shells in the cases of pervious and impervious surfaces, it is convenient to introduce the following non-dimensional variables:

$$
\begin{gathered}
a_{1}=P H^{-1}, a_{2}=Q H^{-1}, a_{3}=R H^{-1}, a_{4}=N H^{-1}, m_{11}=\rho_{11} \rho^{-1}, m_{12}=\rho_{12} \rho^{-1}, m_{22}=\rho_{22} \rho^{-1}, t=\rho_{f} \rho^{-1}, \\
\tilde{x}=\left(V_{0} V_{1}^{-1}\right)^{2}, \tilde{y}=\left(\mathrm{V}_{0} \mathrm{~V}_{2}^{-1}\right)^{2}, \tilde{z}=\left(V_{0} V_{3}^{-1}\right)^{2}, m=V_{f} V_{3}^{-1}, \Omega=\omega h C_{0}^{-1},
\end{gathered}
$$

where $\Omega$ is non-dimensional frequency, $H=P+2 Q+R$, $\rho=\rho_{11}+2 \rho_{12}+\rho_{22}, C_{0}$ and $V_{0}$ are the reference velocities $\left(C_{0}^{2}=\mathrm{N} / \rho, V_{0}^{2}=\mathrm{H} / \rho\right), h$ is the thickness of the poroelastic spherical shell. Let

$$
g=\frac{\mathrm{r}_{2}}{\mathrm{r}_{1}}, \text { so that } \frac{h}{r_{1}}=g-1, \frac{h}{r_{2}}=\frac{g-1}{g} .
$$

Employing the non-dimensional parameters defined in Equations (37), (38) and using the relations given in Abramowitz and Stegun [9]

$$
\begin{gathered}
j_{0}(z)=\frac{\sin z}{z}, j_{1}(z)=\frac{\sin z}{z^{2}}-\frac{\cos z}{z}, \quad \text { where elements } C_{\text {ik }} \text { appearing in Equation (39) are } \\
C_{11}=-\frac{1}{T_{1}} \sin \left(T_{1}\right)+\cos \left(T_{1}\right), C_{12}=\frac{1}{T_{1}} \cos \left(T_{1}\right)+\sin \left(T_{1}\right), \\
C_{13}=-\frac{1}{T_{2}} \sin \left(T_{2}\right)+\cos \left(T_{2}\right), C_{14}=\frac{1}{T_{2}} \cos \left(T_{2}\right)+\sin \left(T_{2}\right), C_{15}=\frac{1}{T_{4}} \sin \left(T_{4}\right)-\cos \left(T_{4}\right), \\
C_{21}=\left[\frac{4 a_{4}}{T_{1}}-\left(a_{1}+a_{2}-a_{2} \delta_{1}^{2}-a_{3} \delta_{1}^{2}\right) T_{1}\right] \sin \left(T_{1}\right)-4 a_{4} \cos \left(T_{1}\right), \\
C_{22}=\left[\left(a_{1}+a_{2}-a_{2} \delta_{1}^{2}-a_{3} \delta_{1}^{2}\right) T_{1}-\frac{4 a_{4}}{T_{1}}\right] \cos \left(T_{1}\right)-4 a_{4} \sin \left(T_{1}\right), \\
C_{23}=\left[\frac{4 a_{4}}{T_{2}}-\left(a_{1}+a_{2}-a_{2} \delta_{2}^{2}-a_{3} \delta_{2}^{2}\right) T_{2}\right] \sin \left(T_{2}\right)-4 a_{4} \cos \left(T_{2}\right), \\
C_{24}=\left[\begin{array}{c}
\left.\left(a_{1}+a_{2}-a_{2} \delta_{2}^{2}-a_{3} \delta_{2}^{2}\right) T_{2}-\frac{4 a_{4}}{T_{2}}\right] \cos \left(T_{2}\right)-4 a_{4} \sin \left(T_{2}\right), \mathrm{C}_{25}=-\frac{t \Omega^{2} a_{4}}{(g-1)^{2} T_{4}} \sin \left(T_{4}\right), \\
C_{31}=\left(a_{3} \delta_{1}^{2}-a_{2}\right) T_{1} \sin \left(T_{1}\right), C_{32}=\left(a_{2}-a_{3} \delta_{1}^{2}\right) T_{1} \cos \left(T_{1}\right), \\
C_{33}=\left(a_{3} \delta_{2}^{2}-a_{2}\right) T_{2} \sin \left(T_{2}\right), C_{34}=\left(a_{2}-a_{3} \delta_{2}^{2}\right) T_{2} \cos \left(T_{2}\right), C_{35}=-\frac{t \Omega^{2} a_{4}}{(g-1)^{2} T_{4}} \sin \left(T_{4}\right),
\end{array}\right.
\end{gathered}
$$


$C_{41}, C_{42}, C_{51}, C_{52}=$ Similar expressions as $C_{21}, C_{22}, C_{31}$, $C_{32}$ with $T_{1}$ replaced by $T_{5}$,

$C_{43}, C_{44}, C_{53}, C_{54}=$ Similar expressions as $C_{23}, C_{24}, C_{33}$, $C_{34}$ with $T_{2}$ replaced by $T_{6}$,

$$
C_{45}=0, C_{55}=0 .
$$

Similarly, the frequency equation of radial vibrations of a fluid filled poroelastic spherical shell for an impervious surface in non-dimensional form is

$$
\left|D_{i k}\right|=0 ; i, k=1,2,3,4,5,
$$

Where $D_{i k}=C_{i k} ; i=1,2,4, k=1,2,3,4,5$,

$$
\begin{aligned}
& D_{31}=\left(a_{2}-a_{3} \delta_{1}^{2}\right) T_{1} \sin \left(T_{1}\right)+\left(a_{3} \delta_{1}^{2}-a_{2}\right) T_{1}^{2} \cos \left(T_{1}\right), \\
& D_{32}=\left(a_{3} \delta_{1}^{2}-a_{2}\right) T_{1} \cos \left(T_{1}\right)+\left(a_{3} \delta_{1}^{2}-a_{2}\right) T_{1}^{2} \sin \left(T_{1}\right),
\end{aligned}
$$

$$
\begin{aligned}
& D_{33}=\left(a_{2}-a_{3} \delta_{2}^{2}\right) T_{2} \sin \left(T_{2}\right)+\left(a_{3} \delta_{2}^{2}-a_{2}\right) T_{2}^{2} \cos \left(T_{2}\right), \\
& D_{34}=\left(a_{3} \delta_{2}^{2}-a_{2}\right) T_{2} \cos \left(T_{2}\right)+\left(a_{3} \delta_{2}^{2}-a_{2}\right) T_{2}^{2} \sin \left(T_{2}\right), \\
& D_{35}=\frac{t \Omega^{2} a_{4}}{(g-1)^{2} T_{4}} \sin \left(T_{4}\right)-\frac{t \Omega^{2} a_{4}}{(g-1)^{2}} \cos \left(T_{4}\right),
\end{aligned}
$$

$D_{51}, D_{52}=$ Similar expressions as $D_{31}, D_{32}$ with $T_{1}$ replaced by $T_{5}$,

$D_{53}, D_{54}=$ Similar expressions as $D_{33}, D_{34}$ with $T_{2}$ replaced by $T_{6}$,

$$
D_{55}=0 \text {. }
$$

The frequency equation of rotatory vibrations of a fluid filled poroelastic spherical shell for a pervious surface Equation (35) in non-dimensional form reduce to

$$
\left|E_{i k}\right|=0, i, k=1,2,3, \cdots 7,
$$

where the elements $E_{i k}$ are

$$
\begin{aligned}
& E_{11}=\left(1-\frac{2}{T_{1}^{2}}\right) \sin \left(T_{1}\right)+\frac{2}{T_{1}} \cos \left(T_{1}\right), E_{12}=\left(\frac{2}{T_{1}^{2}}-1\right) \cos \left(T_{1}\right)+\frac{2}{T_{1}} \sin \left(T_{1}\right), \\
& E_{13}=\left(1-\frac{2}{T_{2}^{2}}\right) \sin \left(T_{2}\right)+\frac{2}{T_{2}} \cos \left(T_{2}\right), \quad E_{14}=\left(\frac{2}{T_{2}^{2}}-1\right) \cos \left(T_{2}\right)+\frac{2}{T_{2}} \sin \left(T_{2}\right), \\
& E_{15}=\frac{2}{T_{3}} \cos \left(T_{3}\right)-\frac{2}{T_{3}^{2}} \sin \left(T_{3}\right), E_{16}=\frac{2}{T_{3}} \sin \left(T_{3}\right)+\frac{2}{T_{3}^{2}} \cos \left(T_{3}\right), \\
& E_{17}=\left(\frac{2}{T_{4}^{2}}-1\right) \sin \left(T_{4}\right)-\frac{2}{T_{4}} \cos \left(T_{4}\right), \\
& E_{21}=\left[\frac{12 a_{4}}{T_{1}^{2}}-\left(4 a_{4}+a_{1}+a_{2}-a_{2} \delta_{1}^{2}-a_{3} \delta_{1}^{2}\right)\right] \sin \left(T_{1}\right)+\left[\left(a_{1}+a_{2}-a_{2} \delta_{1}^{2}-a_{3} \delta_{1}^{2}\right) T_{1}-\frac{12 a_{4}}{T_{1}}\right] \cos \left(T_{1}\right), \\
& E_{22}=\left[\left(4 a_{4}+a_{1}+a_{2}-a_{2} \delta_{1}^{2}-a_{3} \delta_{1}^{2}\right)-\frac{12 a_{4}}{T_{1}^{2}}\right] \cos \left(T_{1}\right)+\left[\left(a_{1}+a_{2}-a_{2} \delta_{1}^{2}-a_{3} \delta_{1}^{2}\right) T_{1}-\frac{12 a_{4}}{T_{1}}\right] \sin \left(T_{1}\right) \text {, } \\
& E_{23}=\left[\frac{12 a_{4}}{T_{2}^{2}}-\left(4 a_{4}+a_{1}+a_{2}-a_{2} \delta_{2}^{2}-a_{3} \delta_{2}^{2}\right)\right] \sin \left(T_{2}\right)+\left[\left(a_{1}+a_{2}-a_{2} \delta_{2}^{2}-a_{3} \delta_{2}^{2}\right) T_{2}-\frac{12 a_{4}}{T_{2}}\right] \cos \left(T_{2}\right), \\
& E_{24}=\left[\left(4 a_{4}+a_{1}+a_{2}-a_{2} \delta_{2}^{2}-a_{3} \delta_{2}^{2}\right)-\frac{12 a_{4}}{T_{2}^{2}}\right] \cos \left(T_{2}\right)+\left[\left(a_{1}+a_{2}-a_{2} \delta_{2}^{2}-a_{3} \delta_{2}^{2}\right) T_{2}-\frac{12 a_{4}}{T_{2}}\right] \sin \left(T_{2}\right) \text {, } \\
& E_{25}=\left(\frac{12 a_{4}}{T_{3}^{2}}-4 a_{4}\right) \sin \left(T_{3}\right)-\frac{12 a_{4}}{T_{3}} \cos \left(T_{3}\right), E_{26}=\left(4 a_{4}-\frac{12 a_{4}}{T_{3}^{2}}\right) \cos \left(T_{3}\right)-\frac{12 a_{4}}{T_{3}} \sin \left(T_{3}\right), \\
& E_{27}=\frac{t \Omega^{2} a_{4}}{(g-1)^{2} T_{4}} \cos \left(T_{4}\right)-\frac{t \Omega^{2} a_{4}}{(g-1)^{2} T_{4}^{2}} \sin \left(T_{4}\right), \\
& \mathrm{E}_{31}=\left(\frac{6 a_{4}}{T_{1}^{2}}-2 a_{4}\right) \sin \left(\mathrm{T}_{1}\right)-\frac{6 a_{4}}{T_{1}} \cos \left(T_{1}\right), \mathrm{E}_{32}=\left(2 a_{4}-\frac{6 a_{4}}{T_{1}^{2}}\right) \cos \left(\mathrm{T}_{1}\right)-\frac{6 a_{4}}{T_{1}} \sin \left(T_{1}\right), \\
& E_{33}=\left(\frac{6 a_{4}}{T_{2}^{2}}-2 a_{4}\right) \sin \left(T_{2}\right)-\frac{6 a_{4}}{\mathrm{~T}_{2}} \cos \left(T_{2}\right), E_{34}=\left(2 a_{4}-\frac{6 a_{4}}{T_{2}^{2}}\right) \cos \left(T_{2}\right)-\frac{6 a_{4}}{T_{2}} \sin \left(T_{2}\right),
\end{aligned}
$$




$$
\begin{gathered}
E_{35}=\left(\frac{6 a_{4}}{T_{3}^{2}}-3 a_{4}\right) \sin \left(T_{3}\right)+\left(a_{4} T_{3}-\frac{6 a_{4}}{T_{3}}\right) \cos \left(T_{3}\right), E_{36}=\left(3 a_{4}-\frac{6 a_{4}}{T_{3}^{2}}\right) \cos \left(T_{3}\right)+\left(a_{4} T_{3}-\frac{6 a_{4}}{T_{3}}\right) \sin \left(T_{3}\right), E_{37}=0, \\
E_{41}=\left(a_{3} \delta_{1}^{2}-a_{2}\right) \sin \left(T_{1}\right)-\left(a_{3} \delta_{1}^{2}-a_{2}\right) \mathrm{T}_{1} \cos \left(T_{1}\right), \mathrm{E}_{41}=\left(a_{2}-a_{3} \delta_{1}^{2}\right) \cos \left(T_{1}\right)-\left(a_{3} \delta_{1}^{2}-a_{2}\right) T_{1} \sin \left(T_{1}\right), \\
E_{43}=\left(a_{3} \delta_{2}^{2}-\mathrm{V}_{2}\right) \sin \left(T_{2}\right)-\left(a_{3} \delta_{2}^{2}-a_{2}\right) T_{2} \cos \left(T_{2}\right), E_{44}=\left(a_{2}-a_{3} \delta_{2}^{2}\right) \cos \left(T_{2}\right)-\left(a_{3} \delta_{2}^{2}-a_{2}\right) T_{2} \sin \left(T_{2}\right), \\
E_{45}=0, E_{46}=0, E_{47}=\frac{t \Omega^{2} a_{4}}{(g-1)^{2} T_{4}} \cos \left(T_{4}\right)-\frac{t \Omega^{2} a_{4}}{(g-1)^{2} T_{4}^{2}} \sin \left(T_{4}\right),
\end{gathered}
$$

$E_{51}, E_{52}, E_{61}, E_{62}, E_{71}, E_{72}=$ Similar expressions as $E_{21}$, $E_{22}, E_{31}, E_{32}, E_{41}, E_{42}$ with $T_{1}$ replaced by $T_{5}$,

$E_{53}, E_{54}, E_{63}, E_{64}, E_{73}, E_{74}=$ Similar expressions as $E_{23}$, $E_{24}, E_{33}, E_{34}, E_{43}, E_{44}$ with $T_{2}$ replaced by $T_{6}$,

$E_{55}, E_{56}, E_{65}, E_{66}=$ Similar expressions as $E_{25}, E_{26}, E_{35}$, $E_{36}$ with $T_{3}$ replaced by $T_{7}$,

$$
E_{57}=0, E_{67}=0, E_{75}=0, E_{76}=0, E_{77}=0 .
$$

Similarly, the frequency equation of rotatory vibrations of a fluid filled poroelastic spherical shell for an impervious surface Equation (36) in non-dimensional form reduce to

$$
\left|F_{i k}\right|=0, i, k=1,2,3 \cdots 7,
$$

where

$$
\begin{gathered}
F_{i k}=E_{i k} ; i=1,2,3,5,6, k=1,2,3,4,5,6,7, \\
F_{41}=\left(a_{3} \delta_{1}^{2}-a_{2}\right)\left(T_{1}^{2}-2\right) \sin \left(T_{1}\right)+2\left(a_{3} \delta_{1}^{2}-a_{2}\right) T_{1} \cos \left(T_{1}\right), \\
F_{42}=\left(a_{3} \delta_{1}^{2}-a_{2}\right)\left(2-T_{1}^{2}\right) \cos \left(T_{1}\right)+2\left(a_{3} \delta_{1}^{2}-a_{2}\right) T_{1} \sin \left(T_{1}\right), \\
F_{43}=\left(a_{3} \delta_{2}^{2}-a_{2}\right)\left(T_{2}^{2}-2\right) \sin \left(T_{2}\right)+2\left(a_{3} \delta_{2}^{2}-a_{2}\right) T_{2} \cos \left(T_{2}\right), \\
F_{44}=\left(a_{3} \delta_{2}^{2}-a_{2}\right)\left(2-T_{2}^{2}\right) \cos \left(T_{2}\right)+2\left(a_{3} \delta_{2}^{2}-a_{2}\right) T_{2} \sin \left(T_{2}\right), \\
F_{45}=0, F_{46}=0, \\
F_{47}=\frac{t \Omega^{2} a_{4}}{(g-1)^{2}}\left(\frac{2}{T_{4}^{2}}-1\right) \sin \left(T_{4}\right)-\frac{2 t \Omega^{2} a_{4}}{(g-1)^{2} T_{4}} \cos \left(T_{4}\right),
\end{gathered}
$$

$F_{71}, F_{72}=$ Similar expressions as $E_{41}, E_{42}$ with $T_{1}$ replaced by $T_{5}$,

$F_{73}, F_{74}=$ Similar expressions as $E_{43}, E_{44}$ with $T_{2}$ replaced by $T_{6}$,

$$
F_{75}=0, F_{76}=0, F_{77}=0 .
$$

In Equations (40), (42), (44) and (46) the quantities $T_{1}$, $T_{2}, T_{3}, T_{4}, T_{5}, T_{6}, T_{7}$ are

$$
T_{1}=\frac{\Omega\left(a_{4} \tilde{x}\right)^{\frac{1}{2}}}{(g-1)}, T_{2}=\frac{\Omega\left(a_{4} \tilde{y}\right)^{\frac{1}{2}}}{(g-1)}, T_{3}=\frac{\Omega\left(a_{4} \tilde{z}\right)^{\frac{1}{2}}}{(g-1)}, T_{4}=\frac{\Omega\left(a_{4} \tilde{z}\right)^{\frac{1}{2}}}{m(g-1)},
$$

$$
T_{5}=\frac{\Omega\left(a_{4} \tilde{x}\right)^{\frac{1}{2}} g}{(g-1)}, T_{6}=\frac{\Omega\left(a_{4} \tilde{y}\right)^{\frac{1}{2}} g}{(g-1)}, T_{7}=\frac{\Omega\left(a_{4} \tilde{z}\right)^{\frac{1}{2}} g}{(g-1)},
$$

and $\delta_{1}^{2}, \delta_{2}^{2}$ in non-dimensional form are

$$
\begin{aligned}
& \delta_{1}^{2}=\left[\left(a_{3} m_{11}-a_{2} m_{12}\right)-\left(a_{1} a_{3}-a_{2}^{2}\right) \tilde{\mathrm{x}}\right]\left[a_{3} m_{12}-a_{2} m_{22}\right]^{-1}, \\
& \delta_{2}^{2}=\left[\left(a_{3} m_{11}-a_{2} m_{12}\right)-\left(a_{1} a_{3}-a_{2}^{2}\right) \tilde{\mathrm{y}}\right]\left[a_{3} m_{12}-a_{2} m_{22}\right]^{-1} .
\end{aligned}
$$

\section{Numerical Results and Discussion}

Two types of poroelastic materials are considered to find the frequency as a function of ratio of thickness of spherical shell to inner radius. One poroelastic material is sandstone saturated with kerosene designated as Material-I [10]. The other poroelastic material is sandstone saturated with water, Material-II [11]. The non-dimensional physical parameters of Material-I and II are given in Table 1.

Frequency Equations (39), (41), (43), (45) and the corresponding frequency equations of empty poroelastic spherical shells each for a pervious and impervious surface constitute a relation between non-dimensional frequency $\Omega$ and ratio of thickness of the poroelastic spherical shell to inner radius $\mathrm{h} / \mathrm{r}_{1}$. When the values of $\mathrm{h} / \mathrm{r}_{1}$ are small it represents thin poroelastic spherical shell. Thickness of the poroelastic spherical shell increases with the increase of $h / r_{1}$. As $h / \mathrm{r}_{1} \rightarrow \infty$ or $r_{1} \rightarrow 0$, empty poroelastic spherical shell becomes a poroelastic solid sphere. To compute the frequency of fluid filled and empty poroelastic spherical shells values of $h / r_{1}$ are taken in the interval $[0.5,7]$. These values of $h / r_{1}$ represents transition of spherical shell from a thin spherical shell to moderately thick shell and then to thick spherical shell. For fluid-filled poroelastic spherical shells, the values of $\mathrm{m}$ and $\mathrm{t}$ are taken as $m=1.5$ and $t=0.4$.

Table 1. Material Parameters.

\begin{tabular}{ccccccccccc}
\hline Material/Parameter & $a_{1}$ & $a_{2}$ & $a_{3}$ & $a_{4}$ & $m_{11}$ & $m_{12}$ & $m_{22}$ & $\tilde{x}$ & $\tilde{y}$ & $\tilde{z}$ \\
\hline I & 0.843 & 0.065 & 0.028 & 0.234 & 0.901 & -0.001 & 0.101 & 0.999 & 4.763 & 3.851 \\
II & 0.960 & 0.006 & 0.028 & 0.412 & 0.877 & 0 & 0.123 & 0.913 & 4.347 & 2.129 \\
\hline
\end{tabular}


Non-dimensional frequency of radial vibrations $(n=0)$ as a function of ratio of thickness to inner radius of a fluid-filled and an empty poroelastic spherical shell is presented in Figure 1 for Material-I each for a pervious and an impervious surface.

From Figure 1 it is clear that frequency of a fluid filled shell for pervious surface decreases in case of a thin spherical shell. As the thickness of the spherical shell increases the frequency increases gradually for moderately thick spherical shell. Still with the increase of thickness of the shell, the frequency remains constant. The frequency of fluid filled spherical shell decreases for thin shell in case of an impervious surface also and it is slightly higher than the frequency of a pervious surface. Then, as the thickness of the spherical shell increases the frequency increases rapidly and for moderately thick shell it remains constant. With the increase of thickness of the shell, the frequency decreases and then remains constant. The frequency of an impervious surface is always higher than that of a pervious surface in case of a fluid filled spherical shell. The frequency of an empty spherical shell slowly increases with the increase of the thickness and for thick spherical shell it is constant. The frequency of an empty spherical thick shell is higher than that of a fluid filled thick shell. This is not true in case of a thin spherical shell. Thus it is seen that the presence of fluid is decreasing the frequency of thick spherical shell. The frequency of an empty spherical shell for an impervious surface is almost same as that of fluid filled moderately thick shell. The frequency of fluid filled and empty thick shells is same. The frequency of an imper- vious surface is higher than that of a pervious surface in case of empty spherical shell also. Thus the frequency of an impervious surface is higher than that of a pervious surface for an empty and a fluid filled spherical shell.

The variation of frequency of radial vibrations of a fluid filled and an empty poroelastic spherical shell each for a pervious an impervious surface is presented in Figure 2 for Material-II. The variation of frequency of fluid filled shell is similar as discussed in Figure 1. But the frequency of empty spherical shell for a pervious an impervious surface is nearly same for thin and moderately thick spherical shells. The frequency of an impervious surface is higher than that of a pervious surface in case of thick empty spherical shells. Presence of mass-coupling parameter has no significant effect on the frequency of fluid filled spherical shell in case of a pervious surface.

The absence of mass-coupling parameter in Material-II is increasing the frequency of an impervious surface for fluid filled spherical shells. This phenomenon is reversed in case of empty poroelastic spherical shells where the presence of mass-coupling parameter is increasing the frequency of an impervious surface. Also it is seen that the presence of fluid is decreasing the frequency in case of a pervious surface while it is increasing the frequency in case of an impervious surface for both the materials.

Frequency of rotatory vibrations $(n=1)$ of fluid filled and empty spherical shells each for a pervious and an impervious surface is presented in Figures 3 and $\mathbf{4}$ for Material-I and Material-II, respectively. From Figure 3 it is clear that the frequency of a fluid filled spherical shell

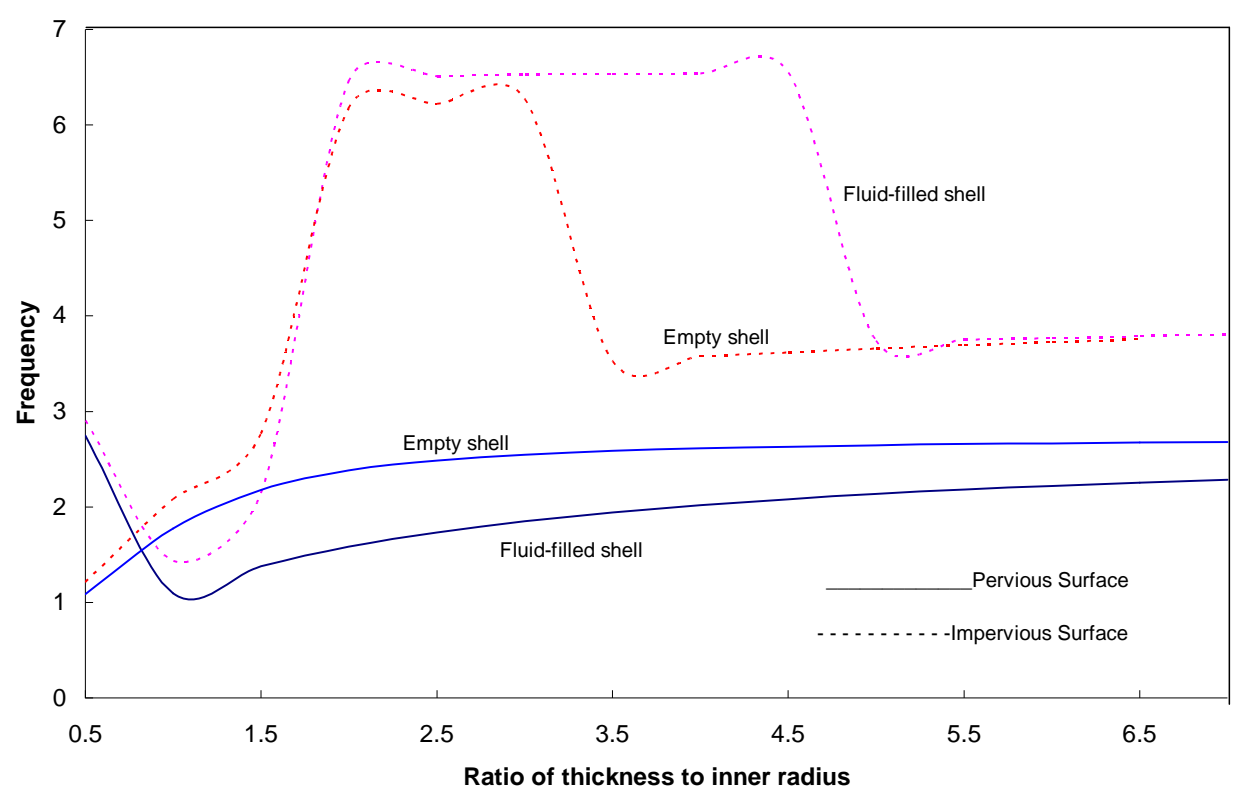

Figure 1. Frequency as a function of ratio of thickness to inner radius Radial vibrations of Fluid-filled and empty spherical shells (Material- I , $n=0$ ). 


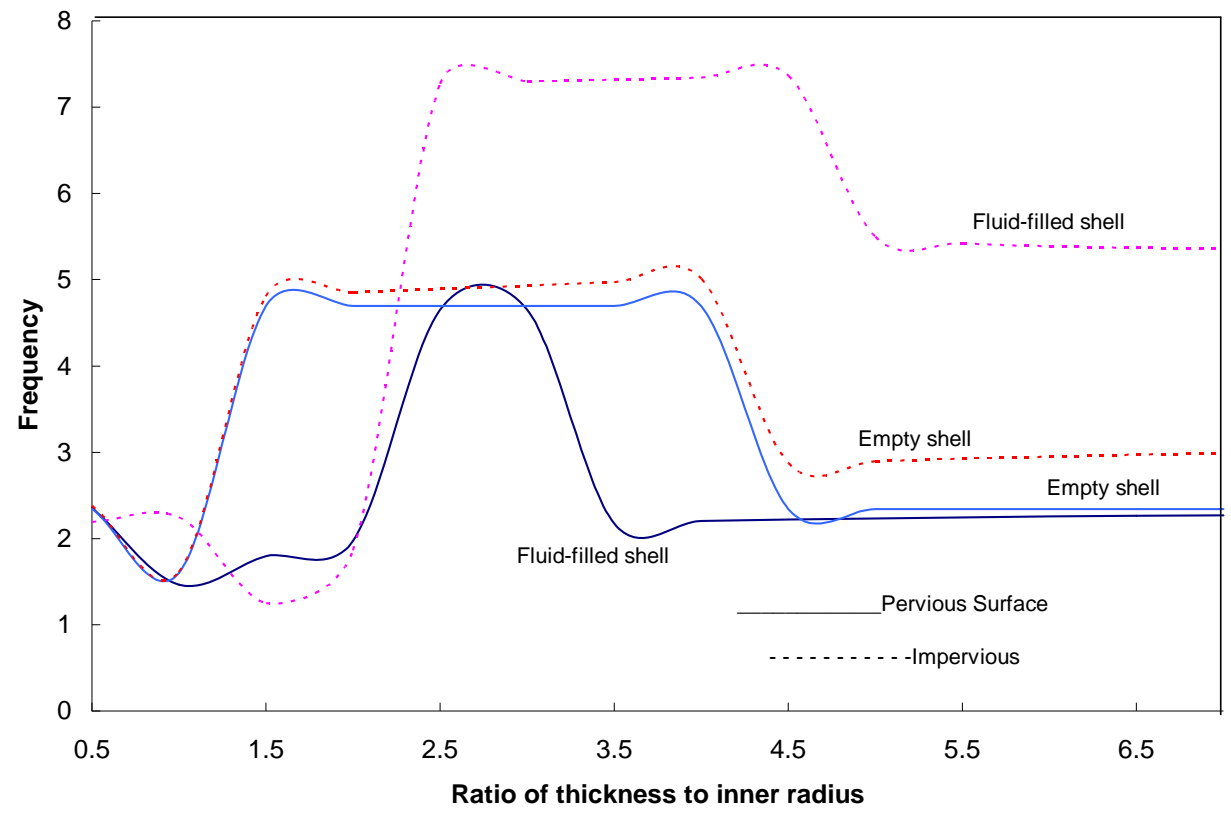

Figure 2. Frequency as a function of ratio of thickness to inner radius Radial vibrations of Fluid-filled and empty spherical shells (Material- II, $\boldsymbol{n}=\mathbf{0}$ ).

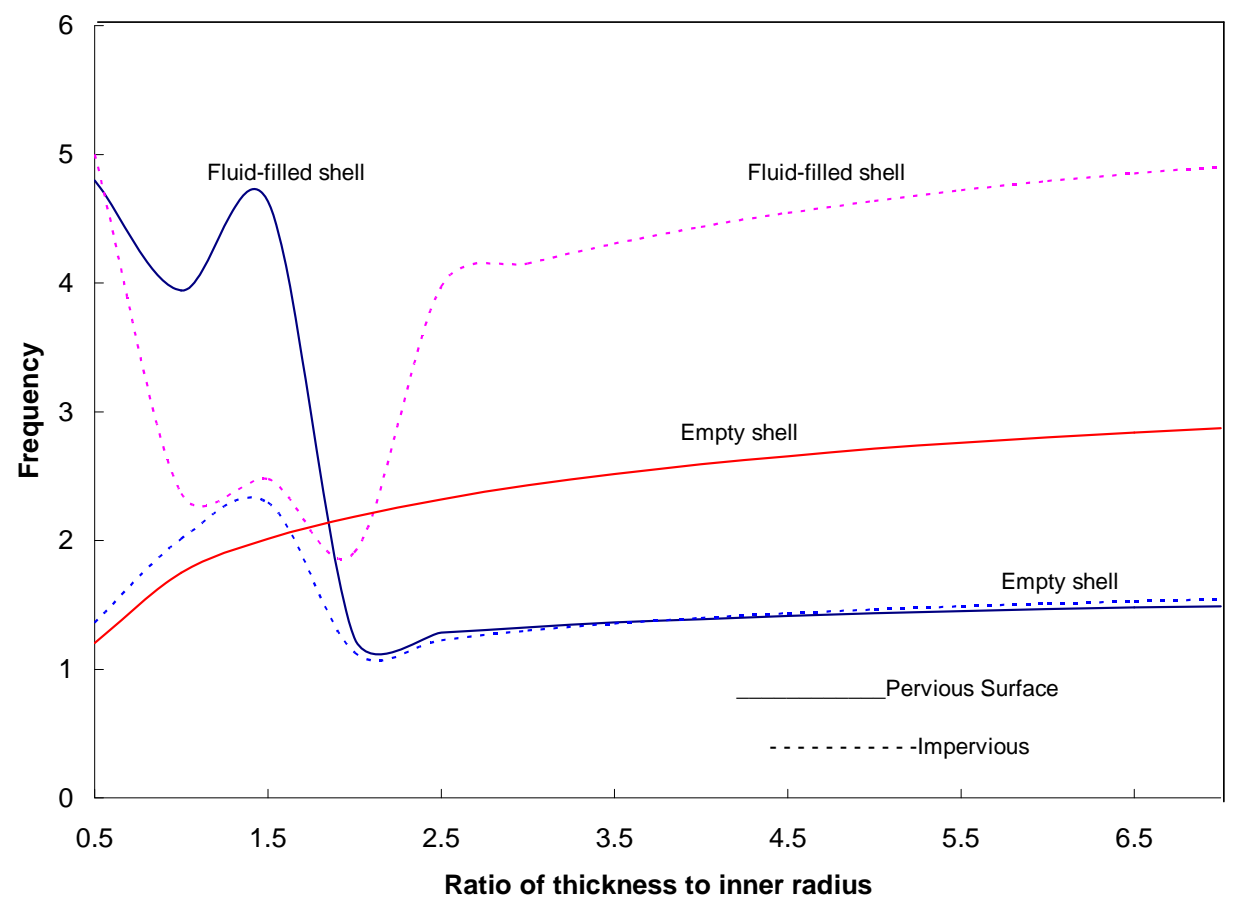

Figure 3. Frequency as a function of ratio of thickness to inner radius Rotatory vibrations of Fluid-filled and empty spherical shells (Material- I , $n=1$ ).

varies in a staggered way when the thickness of the spherical shell is small. With the increase of thickness it remains almost constant. Frequency of thin shell is higher than that of the frequency of moderately thick shell and thick shell in case of a fluid filled shell. The frequency of an impervious surface varies in staggered form for small thickness of the fluid filled shell. But here with the increase of thickness the frequency increases. Thus the frequency of thick shell is higher than the frequency of thin shell in case of fluid filled spherical shell. 


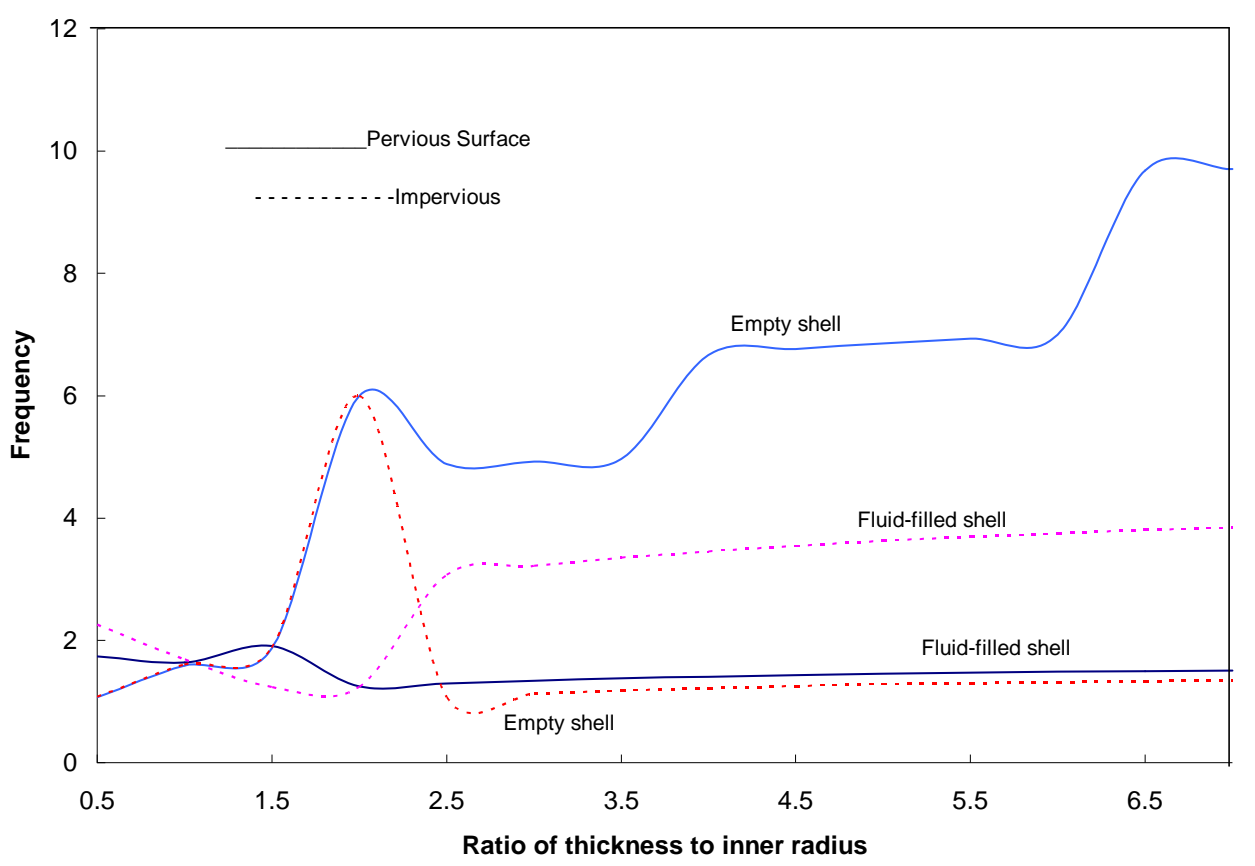

Figure 4. Frequency as a function of ratio of thickness to inner radius Rotatory vibrations of Fluid-filled and empty spherical shells (Material- II, $n=1$ ).

Obviously, the frequency of an impervious surface is higher than the frequency of pervious surface for thick fluid filled spherical shells. Therefore, the presence of fluid is increasing the frequency of an impervious surface for thick shell. The frequency of an empty spherical shell increases with the increase of thickness for a pervious surface. Frequency of an empty shell is higher than the frequency of fluid filled shell. Thus the presence of fluid is decreasing the frequency of a fluid filled shell for a pervious surface. Frequency of an impervious surface of empty spherical shell is lower than the frequency of a fluid filled shell. Thus the absence of fluid in the spherical shell is decreasing the frequency of an empty shell in case of an impervious surface for thick shell.

Figure 4 show the frequency of fluid filled and empty spherical shell each for a pervious an impervious surface in case of Material-II. Frequency of pervious and impervious surface is almost same for thin fluid filled shell.

With the increase of thickness, the frequency of an impervious surface is higher than the frequency of a pervious surface. In case of empty thin spherical shells, again the frequency of pervious and impervious surface is same. With the increase of thickness, the frequency of a pervious surface is higher than the frequency of an impervious surface. It is seen that the presence of masscoupling parameter has no significant effect in case of thick fluid filled shell for a pervious surface. In case of an impervious surface, the presence of mass- coupling parameter is increasing the frequency of fluid filled thick spherical shells. This phenomenon is reversed for empty spherical shells where the absence of mass-coupling parameter is increasing the frequency of a pervious surface while it has no effect on an impervious surface for thick empty shells.

\section{Concluding Remarks}

The study of radial and rotatory vibrations of fluid-filled and empty poroelastic spherical shells has lead to following conclusions:

1) The frequency of an impervious surface in case of radial vibrations is higher than that of a pervious surface in case of a fluid filled and empty spherical shells.

2) Mass-coupling parameter has no significant effect on the frequency of fluid filled spherical shell for a pervious surface in case of radial vibrations.

3) The absence of mass-coupling parameter in Material-II is increasing the frequency of an impervious surface of fluid filled spherical shells in case of radial vibrations.

4) Presence of fluid is increasing the frequency of an impervious surface of thick spherical shell in case of rotatory vibrations.

5) Presence of fluid is decreasing the frequency of a pervious surface for rotatory vibrations.

6) Absence of fluid in the thick spherical shell is decreasing the frequency of an impervious surface for rotatory vibrations. 
7) Mass-coupling parameter is increasing the frequency of fluid filled thick spherical shells of an impervious surface in case of rotatory vibrations.

\section{Acknowledgements}

The authors are thankful to the editor, reviewers and the editorial assistant for their suggestions and kind cooperation in improving the quality of this paper.

\section{References}

[1] R. Kumar, "Axially Symmetric Vibrations of a FluidFilled Spherical Shell," Acustica, Vol. 21, 1969, pp. 143149.

[2] R. Rand and F. DiMaggio, "Vibrations of Fluid Filled Spherical and Spheroidal Shells," Journal of the Acoustical Society of America, Vol. 42, No. 6, 1967, pp. 12781286. doi:10.1121/1.1910717

[3] M. A. Biot, "Theory of Propagation of Elastic Waves in Fluid-Saturated Porous Solid," Journal of the Acoustical Society of America, Vol. 28, 1956, pp. 168-178. doi:10.1121/1.1908239

[4] S. Paul, "A Note on the Radial Vibrations of a Sphere of Poroelastic Material," Indian Journal of Pure and Applied Mathematics, Vol. 7, 1976, pp. 469-475.

[5] G. Chao, D. M. J. Smeulders and M. E. H. van Dongen,
"Sock-Induced Borehole Waves in Porous Formations: Theory and Experiments," Journal of the Acoustical Society of America, Vol. 116, No. 2, 2004, pp. 693-702. doi:10.1121/1.1765197

[6] S. Ahmed Shah, "Axially Symmetric Vibrations of FluidFilled Poroelastic Circular Cylindrical Shells," Journal of Sound and Vibration, Vol. 318, No. 1-2, 2008, pp. 389405. doi:10.1016/j.jsv.2008.04.012

[7] J. N. Sharma and N. Sharma, "Three Dimensional Free Vibration Analysis of a Homogeneous Transradially Isotropic Thermoelastic Sphere," Journal of Applied Mechanics - Transactions of the ASME, Vol. 77, No. 2, 2010, p. 021004.

[8] S. Ahmed Shah and M. Tajuddin, "Torsional Vibrations of Poroelastic Prolate Spheroids," International Journal of Applied Mechanics and Engineering, Vol. 16, 2011, pp. 521-529.

[9] A. Abramowitz and I. A. Stegun, "Handbook of Mathematical Functions," National Bureau of Standards, Washington, 1965.

[10] I. Fatt, "The Biot-Willis Elastic Coefficients for a Sandstone," Journal of Applied Mechanics, Vol. 26, 1959, pp. 296-296.

[11] C. H. Yew, and P. N. Jogi, "Study of Wave Motions in Fluid-Saturated Porous Rocks," Journal of the Acoustical Society of America, Vol. 60, 1976, pp. 2-8. doi:10.1121/1.381045 